\title{
Ground-state description of quasi-one-dimensional polarons with arbitrary electron-phonon coupling strength
}

\author{
A. Erçelebi and R. T. Senger \\ Department of Physics, Bilkent University, 06533 Ankara, Turkey
}

(Received 6 July 1995)

\begin{abstract}
We consider the interaction of a confined electron with bulk polar-optical phonons in a cylindrical quantum well wire with infinite boundary potential. Expressions for the polaron self-energy and mass are derived within a variational scheme over reasonably broad ranges of the wire radius and the phonon-coupling strength. The formulation is based on the standard canonical transformation of the strong-coupling ansatz and consists of a variationally determined perturbative extension serving for the theory to interpolate in the overall range of the coupling constant. Contrary to the general trend that the electron-phonon interaction is inherently stronger in systems of lower dimensionality, our results indicate that, at weak coupling, the binding energy of the polaron can be smaller and its mass less inertial compared with the bulk case when the wire is made narrow.
\end{abstract}

\section{INTRODUCTION}

Recent developments, in microfabrication technology, such as molecular-beam epitaxy and lithographic deposition, have created a variety of opportunities for the fabrication of synthetic semiconductor structures with reduced dimensionality. Of particular interest is the quantum well wire (QWW) configuration, where the ultimate confinement effects quantize the carrier motion in the directions transverse to its length. Since their early prediction ${ }^{1}$ and subsequent fabrication, ${ }^{2-4}$ there has appeared quite a large interest in phonon-coupling-induced effects and polaronic properties of one dimensionally confined electrons. Some considerable amount of the literature published within this context has been devoted to the interaction of electrons with bulk LO phonons and the study of the relevant polaron properties. ${ }^{5-12}$ The common prediction led by these works is that, in quantum wires where the electrons are fundamentally quasi-onedimensional (Q1D), the polaronic binding is far much deeper than in two dimensionally confined quantum well systems. Alternatively stating, high degrees of confinement (as realized in thin wires) lead to a pseudoenhancement in the effective electron-phonon coupling, which in turn brings about the possibility that, in spite of weak polar coupling as in GaAs, for instance, the polaron problem may show up as a strongcoupling aspect coming from confinement effects. This salient feature can be more prominent in II-VI compound semiconductors (e.g., CdTe), where the relevant coupling strengths are almost an order of magnitude larger than those in III-V materials. We thus feel that, for not too weak and pseudoenhanced electron-phonon interaction, the strongcoupling polaron theory, though not capable of reflecting a totally dependable quantitative description, may serve so to provide some qualitative insight into the study of polarons in confined media consisting of materials of somewhat strong polar crystals. On the other hand, a pure perturbation treatment may also be not perfectly appropriate, except for too weak-phonon coupling. We are, therefore, tempted to formulate the Q1D-polaron problem within the framework of a more convenient approach accounting for its weak- and strong-coupling counterparts simultaneously. The formalism that we follow in this work consists of the usage of a perturbative-variational approach used previously by Devreese et al., ${ }^{13}$ in their application to the bulk-optical polaron bound to a Coulomb center. The procedure is structured on basing the starting ansatz on the standard displaced oscillator transformation of the Pekar-strong-coupling theory ${ }^{14}$ and then modifying the adiabatic polaron state by a variationally determined perturbative extension serving for the theory to interpolate in the overall range of the coupling constant.

For the present, we refrain from including the coupling of the electron to the confined phonon modes, as well as interface surface-optical (SO) phonons and adopt the so-called bulk-phonon approximation, where a laterally confined electron is thought of as interacting via the Fröhlich Hamiltonian with the bulk LO phonons of the relevant well material. As such, the fundamental approach followed in this work is to take into account only the generic Q1D aspect of the dynamical behavior of the electron confined in a free-standing tubular geometry and leave out all the other effects; thus, focus our concern primarily to give a clear view of solely the bulk-phonon effects. Apart from ignoring the contributions that may come from all other kinds of phonon modes, we also omit the screening effects and further details, such as those due to the nonparabolicity corrections to the electron band or the loss of validity of both the effective-mass approximation and the Fröhlich continuum Hamiltonian in thin microstructures. In view of these simplifying assumptions, we provide a broad interpolating overview to the onepolaron problem consisting of an electron perfectly confined within a cylindrical boundary with infinite potential. In the next section, we give the basic essentials of the variational theory that we adopt in this calculation and derive analytic expressions for the ground-state binding energy and mass of the Q1D polaron. In Sec. III, we present our numerical results over reasonably wide ranges of the wire radius and the electron-phonon interaction strength. 


\section{THEORY}

Scaling energies by the phonon quantum $\hbar \omega_{\mathrm{LO}}$ and lengths by $\left(\hbar / 2 m^{*} \omega_{\mathrm{LO}}\right)^{1 / 2}$, the Hamiltonian of an electron confined in a wire and interacting with the bulk LO phonons is given by

$$
H=-\nabla^{2}+\mathrm{V}(\rho)+\sum_{Q} a_{Q}^{\dagger} a_{Q}+\sum_{Q} \Gamma_{Q}\left(a_{Q} e^{i \vec{Q} \cdot \vec{r}}+a_{Q}^{\dagger} e^{-i \vec{Q} \cdot \vec{r}}\right),
$$

in which $a_{Q}\left(a_{Q}^{\dagger}\right)$ is the phonon annihilation (creation) operator, and $\vec{r}=(\vec{\rho}, z)$ denotes the electron position in cylindrical coordinates. With the normalization volume set to unity for notational convenience, the interaction amplitude is related to the phonon wave vector $\vec{Q}=\left(\vec{q}, q_{z}\right)$ through $\Gamma_{Q}=\sqrt{4 \pi \alpha} / Q$, where $\alpha$ is the dimensionless coupling constant. We assume that the electron is perfectly confined to a cylindrical wire with infinite potential boundary at $\rho=R$, and take $V(\rho)=0$ inside the wire.

We set the electron wave function as separable in the transverse and longitudinal coordinates in the form

$$
\Phi_{e}(\vec{\rho}, z)=\varphi(\vec{\rho}) Z(z) e^{i \kappa z},
$$

wherein the exponential factor $e^{i \kappa z}$ (with $\kappa$ to be determined variationally) sets the system in motion, thus enabling one to trace the polaron mass along the length of the wire.

\section{A. Displaced oscillator transformation}

The variational approach that we adopt, in this paper, is based on utilizing the usual canonical transformation of the strong-coupling formalism and then extend the adiabatic polaron state, by including an approximate first order perturbative correction, by which it is possible to interrelate the strong- and weak-coupling counterparts of the coupled electron-phonon system. Regardless of the strength of the coupling constant, the starting step in the foregoing theory is to assume a highly rapid charge density fluctuations for the electron, to which the lattice responds by acquiring a relaxed deformation clothing the entire extent of the electron. The adiabatic polaron ground state thus formed is given through a product ansatz consisting of the electron and phonon parts, i.e.,

$$
\Psi_{g}=\Phi_{e}(\vec{\rho}, z)|0\rangle,
$$

together with the Hamiltonian subjected to the displaced oscillator transformation,

$$
H \rightarrow \widetilde{H}=e^{-S} H e^{S},
$$

where

$$
S=\exp \sum_{Q} u_{Q}\left(\Phi_{e}\right)\left[a_{Q}-a_{Q}^{\dagger}\right]
$$

Here, $u_{Q}\left(\Phi_{e}\right)$ is the lattice variational parameter, which will depend on $\vec{r}$, since it is via this parameter an interrelation establishes between the potential well set up by the lattice polarization and the electron which, in turn, becomes trapped in this well. It then follows that, for each choice of $\Phi_{e}$, there is an optimal fit to $u_{Q}$ and, therefore, the transformed Hamiltonian depends on $\Phi_{e}$ implicitly.

Under the transformation (4), the Hamiltonian conforms to

$$
\begin{aligned}
\widetilde{H} & =-\nabla^{2}+\mathrm{V}(\rho)+\sum_{Q} u_{Q}^{2}-\sum_{Q} \Gamma_{Q} u_{Q}[\exp (i \vec{Q} \cdot \vec{r})+\text { c.c. }] \\
& +\sum_{Q} a_{Q}^{\dagger} a_{Q}+\sum_{Q}\left\{\left[\Gamma_{Q} \exp (i \vec{Q} \cdot \vec{r})-u_{Q}\right] a_{Q}+\text { H.c. }\right\} .
\end{aligned}
$$

Since the Hamiltonian is invariant to translations of the electron together with its concomitant lattice distortion, the total momentum along the wire axis

$$
\Pi_{z}=-i \frac{\partial}{\partial z}+\sum_{Q} q_{z} a_{Q}^{\dagger} a_{Q}
$$

must be conserved. The variation, therefore, requires an optimization of the polaron state $e^{S} \Psi_{g}$, which minimizes $H$ subject to the constraint that $\Pi_{z}$ is a constant of motion. Thus, minimizing the functional

$$
\mathrm{F}\left(\beta, v \mid u_{Q}, \kappa\right) \equiv\left\langle\Psi_{g}\left|e^{-S}\left(H-v \Pi_{z}\right) e^{S}\right| \Psi_{g}\right\rangle,
$$

with respect to $\kappa$ and $u_{Q}$ yields

$$
\kappa=\frac{1}{2} v \text { and } u_{Q}\left(\Phi_{e}\right)=\Gamma_{Q} s_{Q} \rho_{Q},
$$

where

$$
\begin{gathered}
s_{Q}=\left\langle\Phi_{e}\left|\exp \left\{ \pm i\left(\vec{q} \cdot \vec{\rho}+q_{z} z\right)\right\}\right| \Phi_{e}\right\rangle, \\
\rho_{Q}=\left(1-v q_{z}\right)^{-1}
\end{gathered}
$$

in which the Lagrange multiplier $v$ is to be identified as the polaron velocity along the wire axis (see, e.g., Ref. 15). In Eq. (8), the symbol $\beta$ stands for the variational parameter(s) contained in $\Phi_{e}(\vec{\rho}, z)$.

In complete form, with the optimal fits for $\kappa$ and $u_{Q}$ substituted in, the Hamiltonian which we shall be refering hereafter is

$$
\begin{aligned}
\tilde{H}= & -\nabla^{2}+\mathrm{V}(\rho)+\sum_{Q} a_{Q}^{\dagger} a_{Q}+\sum_{Q} \Gamma_{Q}^{2} s_{Q}^{2} \rho_{Q}^{2} \\
& -\sum_{Q} \Gamma_{Q}^{2} s_{Q} \rho_{Q}\left(e^{i \vec{Q} \cdot \vec{r}}+e^{-i \vec{Q} \cdot \vec{r}}\right) \\
& +\sum_{Q} \Gamma_{Q}\left(\eta_{Q} a_{Q}+\eta_{Q}^{*} a_{Q}^{\dagger}\right),
\end{aligned}
$$

where

$$
\eta_{Q}=e^{i \vec{Q} \cdot \vec{r}}-s_{Q} \rho_{Q} .
$$

Similarly, for the total momentum transformed accordingly, $\Pi_{z} \rightarrow e^{-S} \Pi_{z} e^{S}$, we have

$$
\begin{aligned}
\widetilde{\Pi}_{z}= & -i \frac{\partial}{\partial z}+\sum_{Q} q_{z} a_{Q}^{\dagger} a_{Q}+\sum_{Q} \Gamma_{Q}^{2} q_{z} s_{Q}^{2} \rho_{Q}^{2} \\
& -\sum_{Q} \Gamma_{Q} q_{z} s_{Q} \rho_{Q}\left(a_{Q}+a_{Q}^{\dagger}\right) .
\end{aligned}
$$


In what follows, we shall consider the case of a stationary polaron, i.e., take $\left\langle\Psi_{g}\left|\widetilde{\Pi}_{z}\right| \Psi_{g}\right\rangle$ as zero, and thus regard $v$ as a virtual velocity that we retain in our calculations to keep track of the effective mass of the coupled electron-phonon complex.

In the case where the coupling constant is thought to be really strong, the visualization of the problem is relatively simple and a reasonable description of the system can readily be achieved by requiring an optimization of the transformed Hamiltonian $\widetilde{H}$, with respect to the ground state, $\Phi_{e}(\vec{\rho}, z)|0\rangle$, of the polaron. We shall retain the results and discussions pertaining to the large $\alpha$ limit until later and point them out as a special case of the more general results, which we derive in the last section. Here, our concern is to make correspondence with the variational scheme of Devreese et al. ${ }^{13}$ where the adiabatic polaron trial state is modified accordingly, so as to cover the overall range of the coupling strength. For the sake of completeness, in the foregoing two subsections, we choose to include a brief revision of the basic essentials in the variational ansatz advanced in Ref. 13. The major distinction, which sets the present concern apart from that in Ref. 13, is that we confine ourselves to a one dimensionally confined polaron model with a virtual momentum imposed to the coupled electron-phonon complex through the factor $\rho_{Q}$, multiplying the term $s_{Q}$ in the Hamiltonian (12).

\section{B. Variational state for arbitrary $\alpha$}

Regardless of the value of $\alpha$, no matter how small it is, the procedure is still to continue with our considerations from Eq. (12), since with decreasing $\alpha$, the degree of localization of the electron becomes reduced in a significant manner; eventually, $s_{Q}$ tends to zero on the average and, thus, $\widetilde{H}$ converts back to its original form $H$ stripped from the displaced oscillator transformation. In view of this reasoning, one is led to include a first order correction to the trial state (3), with the last term in Eq. (12) treated as a perturbation. Since, at present, we limit ourselves to the case of a stationary polaron, we first would like to bring about an insight into the problem with $\rho_{Q}$ in Eq. (12) set to unity, thereby obtain a means of characterizing the polaron (i.e., calculating the optimal $\Phi_{e}$ and hence the binding energy, for instance) for the case when $v=0$. Thereafter, we shall turn on the velocity to keep trace of the polaron mass under a virtual translation of the electron and the lattice distortion together.

In the perturbation treatment of the Fröhlich interaction, the first nonvanishing contribution to the ground-state energy comes from the term, which is of second order in the interaction amplitude. Correspondingly, the leading correction to the trial state defined through Eqs. (3) and (4) is of first order. The ground state trial wave function for $\widetilde{H}$ and for the constraint that the total momentum $\widetilde{\Pi}_{z}$ be conserved, then becomes extended to

$$
\begin{aligned}
\Psi_{g} \rightarrow \widetilde{\Psi}_{g}= & c \Psi_{g}+\sum_{Q} \Gamma_{Q} \sum_{i}\left|\Psi_{i}\right\rangle \\
& \times \frac{\left\langle\Psi_{i}\left|\left(e^{-i \vec{Q} \cdot \vec{r}}-s_{Q}\right) a_{Q}^{\dagger}\right| \Psi_{g}\right\rangle}{\Delta \varepsilon_{i-g}} .
\end{aligned}
$$

In the above, $c$ is a constant, which serves for normalization, and the index $i$ refers to the intermediate states, those consisting of the electron and one-phonon with wave vector $\vec{Q}$. The summation over the intermediate states is a rather difficult task, since now the states themselves and the corresponding energies depend on $\alpha$ and the lattice coordinates in involved manners. Nevertheless, this shortcoming can be eliminated by replacing the energy denominator $\Delta \varepsilon_{i-g}$ by an average quantity,

$$
g_{Q}=\left\langle\frac{1}{\Delta \varepsilon_{i-g}}\right\rangle_{i}
$$

which in the calculation will be determined variationally. Using completeness, the $i$ summation in Eq. (15) can be projected out to yield ${ }^{13}$

$$
\widetilde{\Psi}_{g}=\left\{c+\sum_{Q} \Gamma_{Q} g_{Q}\left(e^{-i \vec{Q} \cdot \vec{r}}-s_{Q}\right) a_{Q}^{\dagger}\right\} \Psi_{g} .
$$

The variational parameter $g_{Q}$ sets up a fractional admixture of the strong- and weak-coupling counterparts of the coupled electron-phonon system and thus is expected to serve for the theory to interpolate between the extreme limits of the coupling constant.

\section{Formulation}

The requirement that the extended trial state $\widetilde{\Psi}_{g}$ be normalized yet poses a further constraint, interrelating the parameters $c$ and $g_{Q}$ through

$$
f\left(c, g_{Q}\right)=c^{2}+\sum_{Q} \Gamma_{Q}^{2} g_{Q}^{2} h_{Q}-1=0
$$

in which

$$
h_{Q}=\left\langle 0\left|\left(e^{i \vec{Q} \cdot \vec{r}}-s_{Q}\right)\left(e^{-i \vec{Q} \cdot \vec{r}}-s_{Q}\right)\right| 0\right\rangle=1-s_{Q}^{2} .
$$

In order to find the optimal fit to $g_{Q}$, one has to minimize the expectation value of $\widetilde{H}-v \widetilde{\Pi}_{z}$ in the trial state (17), subject to the constraint (18). Within the framework of the modified trial state $\widetilde{\Psi}_{g}$, the functional (8) now takes the form

$$
\begin{aligned}
\mathrm{F}\left(\beta, v \mid c, g_{Q}\right)= & c^{2}\left(e_{0}+\frac{1}{4} v^{2}\right)-\frac{1}{2} v^{2}+\left(1-2 c^{2}\right) \chi \\
& +2 c \sum_{Q} \Gamma_{Q}^{2} g_{Q} h_{Q}+\sum_{Q} \Gamma_{Q}^{2} g_{Q}^{2}\left(e_{Q}-\delta_{Q}+h_{Q}\right),
\end{aligned}
$$

where

$$
\begin{gathered}
e_{0}=\left\langle\Phi_{e}\left|-\nabla^{2}\right| \Phi_{e}\right\rangle, \\
e_{Q}=\left\langle\Phi_{e}\left|\left(e^{i \vec{Q} \cdot \vec{r}}-s_{Q}\right)\left(-\nabla^{2}\right)\left(e^{-i \vec{Q} \cdot \vec{r}}-s_{Q}\right)\right| \Phi_{e}\right\rangle \\
=e_{Q}^{(0)}-v\left(q_{z}-\frac{1}{4} v\right) h_{Q},
\end{gathered}
$$

with

$$
e_{Q}^{(0)}=q^{2}+\frac{1}{2} q_{z}^{2}+\left(e_{0}+\frac{1}{2} q_{z}^{2}\right) h_{Q}
$$


and furthermore,

$$
\begin{gathered}
\chi=\sum_{Q} \Gamma_{Q^{2}}^{2} s_{Q}^{2} \rho_{Q}, \\
\delta_{Q^{\prime}}=\sum_{Q^{\prime}} \Gamma_{Q^{\prime}}^{2} s_{Q^{\prime}} \Delta_{Q Q^{\prime}} \rho_{Q^{\prime}},
\end{gathered}
$$

wherein

$$
\Delta_{Q Q^{\prime}}=\left\langle 0\left|\left(e^{i \vec{Q} \cdot \vec{r}}-s_{Q}\right)\left(e^{i \vec{Q}^{\prime} \cdot \vec{r}}+e^{-i \vec{Q}^{\prime} \cdot \vec{r}}\right)\left(e^{-i \vec{Q} \cdot \vec{r}}-s_{Q}\right)\right| 0\right\rangle .
$$

The variational fit to $g_{Q}$ (and to the normalization constant $c$ ) is achieved by requiring

$$
\frac{\partial}{\partial g_{Q}}\left\{\mathrm{~F}\left(\beta, v \mid c, g_{Q}\right)-\Lambda f\left(c, g_{Q}\right)\right\}=0,
$$

with $\Lambda$ being a Lagrange multiplier. It then follows that the functional $F$ is given by

$$
\mathrm{F}(\beta, v)=e_{0}-\chi-\frac{1}{4} v^{2}+\Lambda
$$

where $\Lambda$ is derived through the transcendental equation,

$$
\Lambda=\sum_{Q} \Gamma_{Q}^{2}\left[g_{Q} / c\right] h_{Q}
$$

in which

$$
\frac{g_{Q}}{c}=-\frac{h_{Q}}{D_{Q}}
$$

and

$$
D_{Q}=e_{Q}-\delta_{Q}+\left(1-e_{0}-\frac{1}{4} v^{2}+2 \chi-\Lambda\right) h_{Q} .
$$

In order to trace out the polaron mass from Eq. (28), we have to split $\mathrm{F}(\beta, v)$ into its parts consisting of the binding energy of the polaron alone and the additional kinetic contribution, which shows up having imposed a virtual momentum to the polaron. We are thus tempted to expand Eqs. (24), (25) and the summand in Eq. (29) in a power series up to second order in $v$. We, therefore, conform $\chi$ and $\delta_{Q}$ into the forms

$$
\chi=\chi^{(0)}+\frac{1}{4} v^{2} \chi^{(1)} \text { and } \delta_{Q}=\delta_{Q}^{(0)}+\frac{1}{4} v^{2} \delta_{Q}^{(1)},
$$

where $\chi^{(n)}$ and $\delta_{Q}^{(n)}(n=0,1)$ are given by

$$
\begin{gathered}
\chi^{(n)}=\sum_{Q} \Gamma_{Q^{2}}^{2} s_{Q}^{2}\left[2 q_{z}\right]^{2 n}, \\
\delta_{Q}^{(n)}=\sum_{Q^{\prime}} \Gamma_{Q^{\prime}}^{2} s_{Q^{\prime}} \Delta_{Q Q^{\prime}}\left[2 q_{z}^{\prime}\right]^{2 n} \\
=2 \chi^{(n)}\left(1+s_{Q}^{2}\right)-2 s_{Q} \sum_{Q^{\prime}} \Gamma_{Q^{\prime}}^{2} s_{Q^{\prime}} \\
\times\left(s_{Q+Q^{\prime}}+s_{Q-Q^{\prime}}\right)\left[2 q_{z}^{\prime}\right]^{2 n},
\end{gathered}
$$

in which $s_{Q \pm Q^{\prime}}$ refers to the same expression as for $s_{Q}$, cf. Eq. (10), in which $\vec{q}$ is to be replaced by $\vec{q} \pm \vec{q}^{\prime}$, and $q_{z}$ by $q_{z} \pm q_{z}^{\prime}$.

Furthermore, setting

$$
D_{Q}^{(0)}=e_{Q}^{(0)}-\delta_{Q}^{(0)}+\left(1-e_{0}+2 \chi^{(0)}-\Lambda\right) h_{Q},
$$

we obtain

$$
\mathrm{F}(\beta, v)=E_{g}(\beta)-\frac{1}{4} v^{2} m_{p},
$$

where

$$
E_{g}(\beta)=e_{0}-\chi^{(0)}+\Lambda
$$

refers to the ground-state energy and the factor $m_{p}$ multiplying $\frac{1}{4} v^{2}$ is identified as the polaron mass given by

$$
\begin{aligned}
m_{p}= & 1+\chi^{(1)}+\sum_{Q} \Gamma_{Q}^{2} \frac{h_{Q}^{2}}{D_{Q}^{(0)}}\left\{\left[2 q_{z} h_{Q} / D_{Q}^{(0)}\right]^{2}\right. \\
& \left.+\left[\delta_{Q}^{(1)}-2 \chi^{(1)} h_{Q}\right] / D_{Q}^{(0)}\right\} .
\end{aligned}
$$

The explicit analytic forms for the quantities $e_{0}, s_{Q}, \chi^{(n)}$, and $\delta_{Q}^{(n)}$, involved in Eqs. (37) and (38), can be derived using the functional form for $\Phi_{e}(\vec{\rho}, z)$, which we introduce in the next section. They are, however, lengthy to write here and therefore, we list them in the Appendix.

It should be clear that, in deriving Eq. (36), we have regarded parameter $\Lambda$ as being obtained from Eq. (29), for $g_{Q} / c=-h_{Q} / D_{Q}^{(0)}$, i.e., for the case where the polaron is taken as stationary.

\section{RESULTS AND CONCLUSIONS}

Due to the analytic complexity, the optimal fits to $\Lambda$ and $\Phi_{e}$ are to be performed by numerical methods within an iterative scheme. In our calculations, we select the electron wave function $\Phi_{e}(\vec{\rho}, z)$, given by Eq. (2) in a reasonably simplest form, where its transverse and longitudinal parts are given by

$$
\varphi(\vec{\rho})=\frac{\mathrm{J}_{0}\left(j_{0,1} \rho / R\right)}{\sqrt{\pi} R \mathrm{~J}_{1}\left(j_{0,1}\right)}
$$

and

$$
Z(z)=\left(\frac{\beta^{2}}{\pi}\right)^{1 / 4} \exp \left(-\frac{1}{2} \beta^{2} z^{2}\right)
$$

Here, $J_{n}$ denotes the $n$th order cylindrical Bessel function of the first kind and $j_{0,1} \approx 2.4048$ is the first zero of $J_{0}$. Parameter $\beta$ is to be adjusted variationally and provides a measure of the spatial extent of the electron along the wire axis, i.e., the root mean square of the coordinate $z$ is related to $\beta$ through 


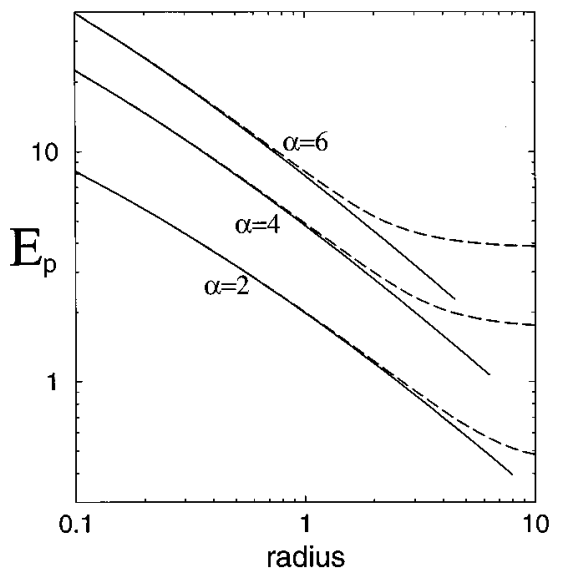

FIG. 1. The binding energy, as a function of the wire radius calculated within the strong coupling theory. The solid and dashed curves are for $\varphi(\vec{\rho})$, taken as given by Eqs. (39) and (42), respectively.

$$
\begin{aligned}
\xi_{z} & =\left\{\left\langle\widetilde{\Psi}_{g}\left|z^{2}\right| \widetilde{\Psi}_{g}\right\rangle\right\}^{1 / 2} \\
& =\frac{1}{\sqrt{2} \beta} \sqrt{\frac{1+\Sigma_{Q} \Gamma_{Q}^{2}\left(g_{Q} / c\right)^{2}\left[1-\left(1-q_{z}^{2} / \beta^{2}\right) s_{Q}^{2}\right]}{1+\Sigma_{Q} \Gamma_{Q}^{2}\left(g_{Q} / c\right)^{2} h_{Q}}} .
\end{aligned}
$$

We think that, for not too large $\alpha$, the choice (39) for $\varphi(\vec{\rho})$ is well suited for thin wire structures, as the transverse localization is provided most dominantly by the wire-boundary potential, rather than the phonon-coupling-induced localization. We could, as well, have chosen $\varphi(\vec{\rho})$ as more general like,

$$
\varphi(\vec{\rho}) \rightarrow \varphi(\vec{\rho}) \exp \left(-\beta^{\prime 2} \rho^{2}\right),
$$

consisting of a Gaussian extension, for instance. This latter wave form duplicates the same features at small wire radii and is expected to give better results in the range of large $R$, and moreover, to depict asymptotically the bulk limit when $R \rightarrow \infty$. However, we still adopt the former expression (39) for $\Phi_{e}$, mainly to facilitate the analytic and numeric computations. In the following we, therefore, restrict our considerations solely to thin wires, rather than bulklike media, where the relevant polaron properties have already been well understood in the literature. In this regard, we shall be content with a comparison of the two wave forms (39) and (42) within only the framework of the strong-coupling approximation with $\alpha$ selected as larger than 1, where the discrepancy is expected to be somewhat more prominent than that in the intermediate and weak-coupling regimes (cf. Fig. 1 ). From the succession of curves for $\alpha=2,4$, and 6, we observe that the energy values, derived from (39), exhibit a considerable amount of digression from what one expects for large $R$, and in particular, the digression grows larger for stronger $\alpha$. We also note that the place at which the curves for (39) and (42) start to get deviated shifts down to smaller $R$ values for stronger phonon coupling, since for large $\alpha$, the polaron is already in a highly localized state and a small sized polaron becomes influenced by the confining boundary

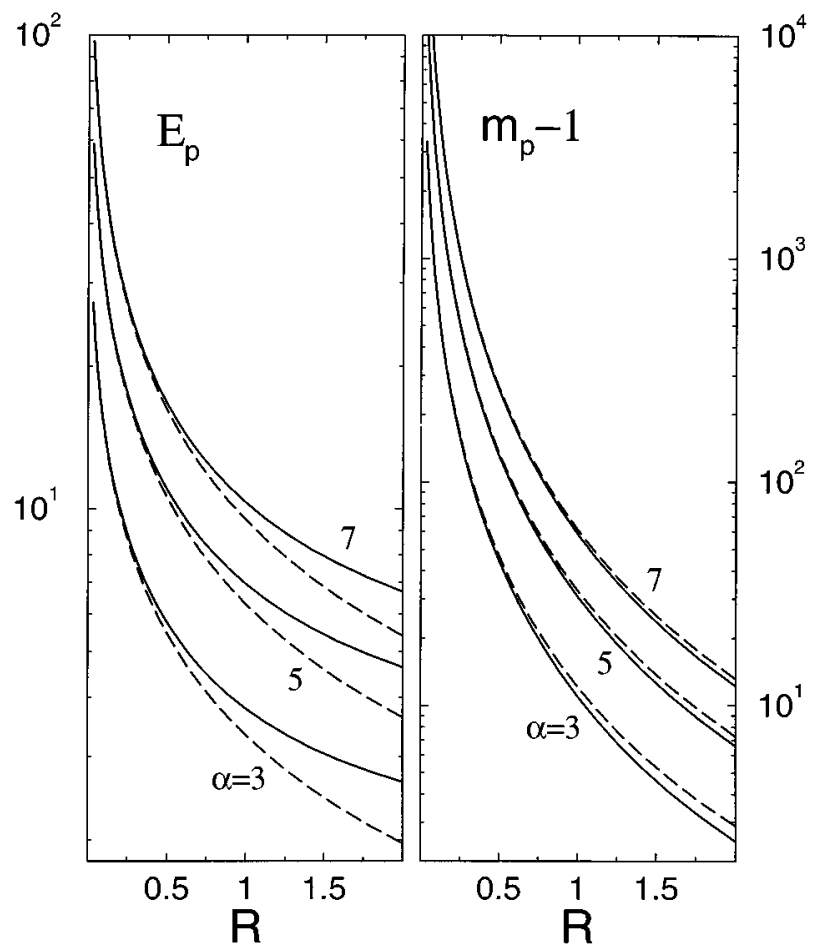

FIG. 2. (a) The binding energy $E_{p}$, and (b) the effective polaron mass $m_{p}$, as a function of the wire radius. The solid and dashed curves reflect the results of the present and strong-coupling approximations, respectively.

only for small wire radius. On the contrary, however, we see that, for not too large $\alpha$, both wave functions, (39) and (42), give almost identical binding energies for $R \leqslant 2$, and that the wave form (39), which we use in our calculations, becomes capable of reflecting a reasonable description of the system over a broader range of $R$, when $\alpha$ is made weaker.

In displaying the results of the present formulation, we first refer to the regime of strong phonon coupling and provide plots of the binding energy $E_{p}=\left(j_{0,1} / R\right)^{2}-E_{g}$ and the polaron mass $m_{p}$ against the wire size for a succession of large $\alpha$ values. An immediate glance at the set of curves in Fig. 2 reveals that, with increasing degree of confinement (i.e., with increasing $R^{-1}$, as well as with increasing $\alpha$ ), the binding becomes substantially deepened, where, correspondingly, the effective polaronic mass scales to large values with very pronounced slopes. Comparing our results with those derived from the strong-coupling theory (cf. dashed curves), we note that the present approach yields significantly improved energy upper bounds and that the strong-coupling approximation deviates considerably from the present formalism as $\alpha$ is made weaker and/or $R$ is increased. Indeed, it is only for large $\alpha$ and small $R$ that the two approaches become identical since, in this limit, the electron gets highly localized, $s_{Q}(10)$ becomes unity on the average, and thus $h_{Q}$ (19), and hence $\Lambda$ (29) tend to zero and the present theory readily reproduces the strong-coupling limit, i.e.,

$$
E_{g}=e_{0}-\chi^{(0)} \text { and } m_{p}=1+\chi^{(1)},
$$

as depicted by the curves (solid and dashed) that become closer and eventually match as the phonon coupling is made stronger and $R$ is tuned to small values. 
Alternatively stating, for not too strong $\alpha$, the pure strong-coupling treatment of the problem is totally inadequate to reflect any weak-coupling aspect and this shortcoming is eliminated in the present approach by solving the transcendental equation (29) for the term $\Lambda$ in the energy expression, since it is only through this term that a detailed interbalance is set up between the strong- and weak-coupling counterparts of the coupled electron-phonon system. As $\alpha$ is shifted down to small values, the role $\Lambda$ plays becomes very prominent, and in case the electron is loosely bound, the polaron binding is mostly determined by this term. In particular, for a reduced degree of confinement $(R \gg 1)$ and at weak coupling ( $\alpha \ll 1)$, it is easy to see that the terms $e_{0}$, $\delta_{Q}, \chi$, and $\Lambda$ in Eq. (31) become far too small to yield any significant contribution to the summand in the trancendental equation (29). Therefore, in Eqs. (37) and (38), retaining only $h_{Q} \approx 1$ and $e_{Q} \approx Q^{2}$, we readily obtain

$$
\begin{aligned}
E_{g} & \approx \Lambda \approx-\sum_{Q} \Gamma_{Q}^{2}\left(1+Q^{2}\right)^{-1}=-(2 / \pi) \alpha \int_{0}^{\infty} d Q\left(1+Q^{2}\right)^{-1} \\
& =-\alpha
\end{aligned}
$$

and

$$
m_{p} \approx 1+4 \sum_{Q} \Gamma_{Q}^{2} q_{z}^{2}\left(1+Q^{2}\right)^{-3}=1+\frac{1}{6} \alpha
$$

which are the well established energy and mass values for the bulk polaron in the weak $\alpha$ limit; thus exemplifying the essential role which $\Lambda$ plays in conforming the adiabatic approximation over to the results derived from the perturbation theory.

An important remark pertaining to a weakly coupled polaron in a narrow wire is that the electron now has to choose between two contrasting aspects of whether to conform to a delocalized state with a correspondingly large spread when $\alpha \ll 1$, or to acquire a localized configuration as the wire is made thinner. It should be mentioned that, the parameters $\alpha$ and $R$ characterizing the system do not enter the problem in an independent way, but together take part in a related manner in the binding, dominating the effect of one another, and yet acting collaboratively in favor of stronger binding. Thus, a high degree of localization in reduced dimensionality is expected to lead to a pseudoenhancement in the effective electron-phonon coupling, which in turn brings about the possibility that, in spite of weak polar coupling, as in compound semiconductors, the polaron binding may as well have a strong-coupling counterpart coming from confinement effects in narrow wires.

As reference to weak electron-phonon coupling, we select CdTe $(\alpha \simeq 0.40)$ and GaAs $(\alpha \simeq 0.07)$ based quantum wires, which are of particular interest as typical examples of II-VI and III-V compound semiconductors. An examination of the curves for CdTe and GaAs in Fig. 3 reveals that both $E_{p}$ and $m_{p}$ undergo rather distinctive types of variations when we vary $R$. We observe that for $\alpha=0.40$; the binding (and hence the mass) become monotonically stronger and more inertial, as the dimensionality is tuned from three dimensional to quasi-one-dimensional. This is totally consistent with what
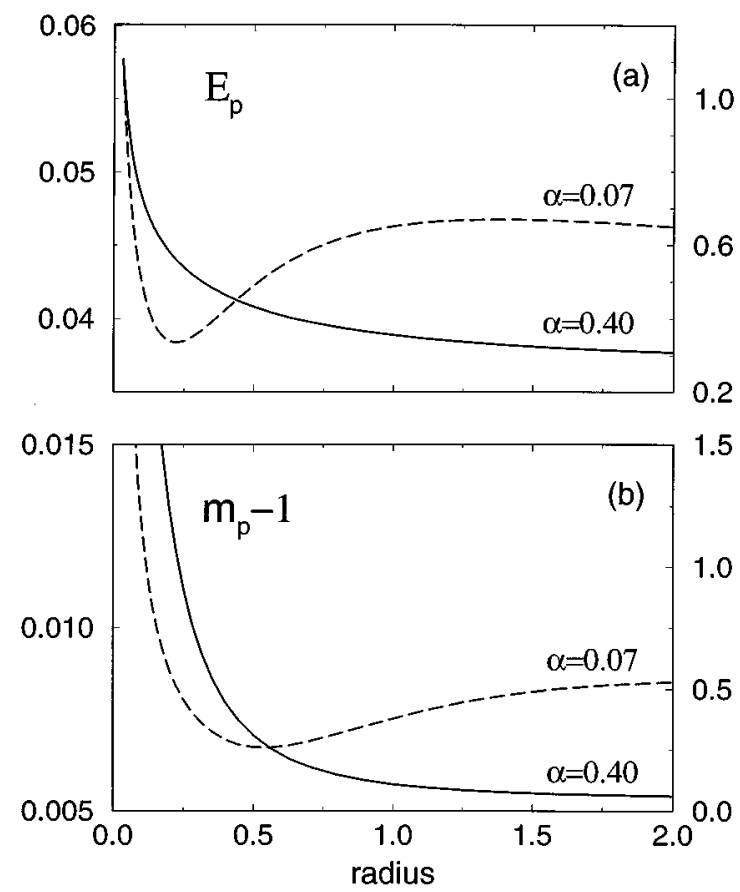

FIG. 3. (a) The binding energy $E_{p}$, and (b) the effective polaron mass $m_{p}$, as a function of the wire radius. The solid and dashed curves are for CdTe and GaAs based quantum wires, respectively. In the plots, the energy and length units correspond, respectively, to 18 (35) $\mathrm{meV}$ and 44 (40) $\AA$, for CdTe (GaAs).

one usually expects for systems of reduced dimensionality, and originates essentially from that, with decreasing $R$, the wave function is squeezed onto the wire axis in all transverse directions, resulting in a reduction in the overall spatial extent of the polaron on the average and hence in the effective dimensionality, thus leading to deeper polaronic binding.

For even weaker coupling (as in GaAs, for instance-cf. the dashed curves in Fig. 3), the behavior is rather different. Beginning from the bulk case and approaching the onedimensional limit, there comes about a competitive interrelation between whether the charge density fluctuations of the electron will condense onto the polaron center or will expand to relax itself in the longitudinal $( \pm z)$ directions along the wire axis. Starting from $R \gg 1$ and then restricting the transverse spread of the electron, the contribution coming from the tendency of the electron to expand longitudinally dominates first, causing a decrease in the binding energy, and correspondingly leads to a smaller effective mass of the polaron. Meanwhile, with contracting a wire radius, the electronic spread experiences an increasingly large restriction towards the wire axis and therefore, below a certain wire size, the effective degree of localization of the electron-phonon system starts to increase, leading to a considerably pronounced effective phonon coupling and hence to deeper polaronic binding. For comparatively stronger $\alpha$, this salient feature becomes less prominent and does not even show up, since the starting state of the system is already a localized one.

In Fig. 4, we provide a global comprehensive summary of the variation of the binding energy as a function of $\alpha$ and $R$. We observe that, regardless of the wire width, $E_{p}$ (and 


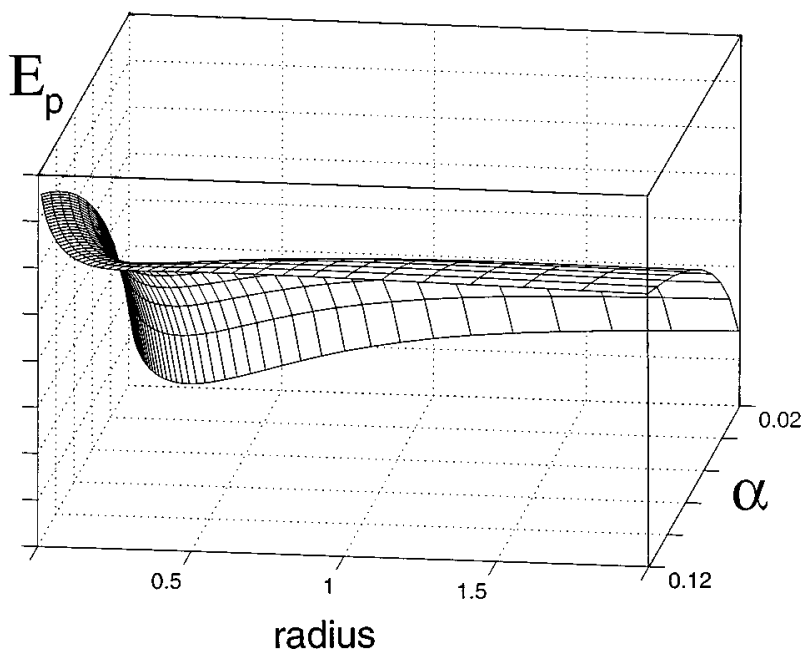

FIG. 4. The binding energy (in arbitrary units on a logarithmic scale), as a function of the coupling constant and the wire radius.

$m_{p}$ - not pictured in the figure) always increase monotonically with increasing $\alpha$. So it is true with decreasing $R$ as well, only however, for $\alpha$ lying above some value around 0.10 . Below this value of $\alpha$, the energy and mass profiles (viewed as a function of decreasing $R$ ) are seen to decrease first, and then increase after going through a minimum as in the aforementioned description given for the GaAs wire. To give somewhat more impact to this interesting type of variation of the polaronic binding, we portray the longitudinal extent of the polaron, $\xi_{z}(41)$, over the relevant range of weak $\alpha$ and the wire radius (cf. Fig. 5). We note that when $\alpha$ is small, $\xi_{z}$ has first a tendency to expand and, after having displayed a peaked profile, shrinks as the wire radius is reduced to smaller values. For large values of $\alpha$, however, $\xi_{z}$ is seen to shrink monotonically without showing any prominent increase, since, in this case, the polaron has already a comparatively deeper self-induced potential, and an increase in the degree of confinement makes the polaron even more deeply bound and more localized.

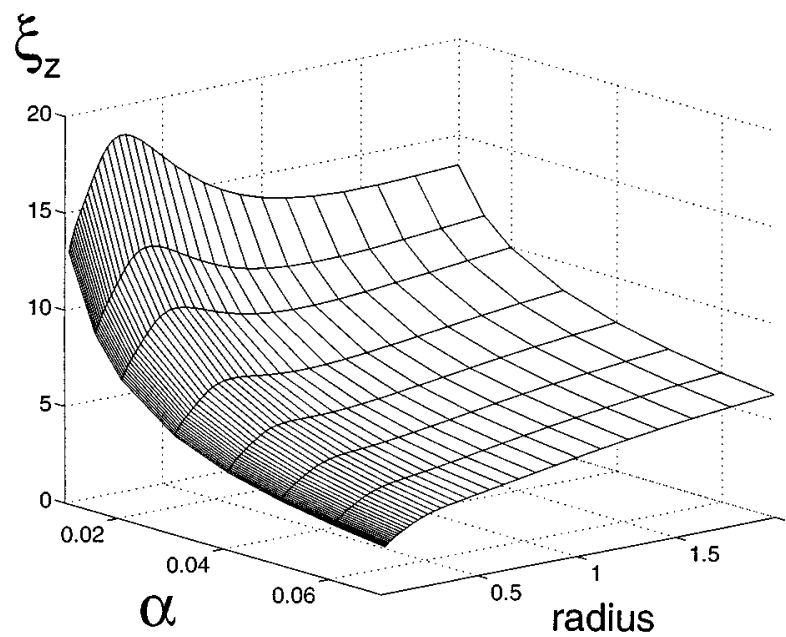

FIG. 5. The longitudinal spatial extent $\xi_{z}$, as a function of the coupling constant and the wire radius.

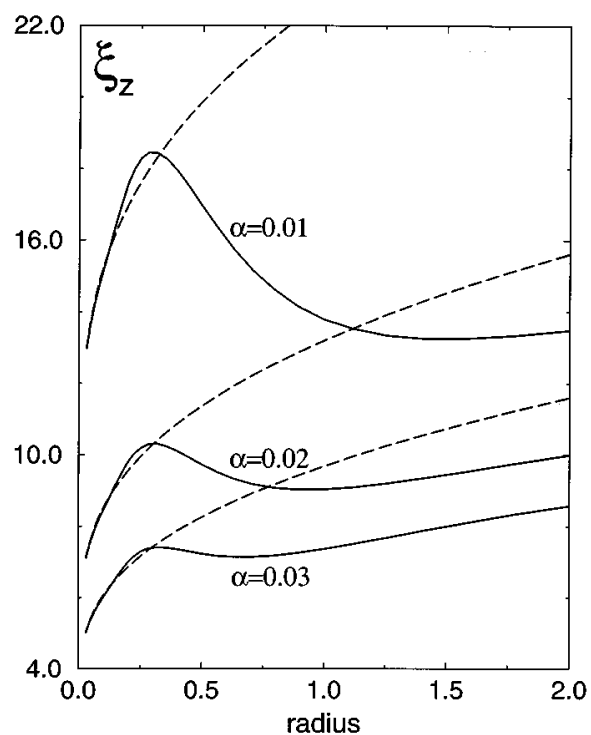

FIG. 6. The longitudinal spatial extent $\xi_{z}$, as a function of the wire radius at weak coupling. The solid and dashed curves reflect the results of the present and strong-coupling approximations, respectively.

An interesting remark pertaining to the regime of weak coupling is that even for coupling constants as small as $\alpha \sim 0.01$, a "pseudostrong-coupling" condition can be reached at high degrees of confinement. For completeness, we exemplify this feature in Fig. 6, where we plot $\xi_{z}$ (calculated from both the strong coupling and present theories) against the wire size. From the succession of the pairs of curves for $\alpha=0.01,0.02$, and 0.03 , we readily note that, at highly confined configurations of the polaron, the $\xi_{z}$ profiles calculated from Eq. (42) are fairly close to those derived from the strong-coupling theory: $\xi_{z}=1 / \sqrt{2} \beta$, where now the optimal $\beta$ is to be obtained by minimizing the energy expression given by Eq. (43). As the confining boundary is made to expand, however, the strong-coupling theory rapidly loses its validity and deviates rather drastically from the present formalism, both in terms of magnitude and qualitative nature; and the digression grows at much faster rates for smaller values of the coupling constant.

In summary, this work revises the ground-state property of the optical polaron confined in a cylindrical quantum wire of infinite boundary potential. The formalism adopted here allows one to trace out the polaron quantities of general interest within an interpolating scheme, accounting for the fractional admixture of the weak- and strong-coupling counterparts of the coupled electron-phonon complex. Contrary to the general trend that the effective electron-phonon coupling is inherently stronger in systems of lower dimensionality, we find that, at weak $\alpha$ and for thin wires, the polaronic binding may get loose and even become weaker than for the bulk case.

\section{APPENDIX}

The functional forms for the quantities $e_{0}, s_{Q}, \chi^{(n)}$, and $\delta_{Q}^{(n)}(n=0,1)$, calculated using Eqs. (39)-(40) are given by 


$$
\begin{gathered}
e_{0}=\left(j_{0,1} / R\right)^{2}+\frac{1}{2} \beta^{2}, \\
s_{Q}=A(q R) \exp \left(-\frac{q_{z}^{2}}{4 \beta^{2}}\right), \\
\chi^{(0)}=\alpha \int_{0}^{\infty} d q A^{2}(q R) e^{\gamma^{2}} \operatorname{erfc}(\gamma), \\
\chi^{(1)=\sqrt{32 / \pi}} \alpha \beta \int_{0}^{\infty} d q q A^{2}(q R)\left\{1-\sqrt{\pi} \gamma e^{\gamma^{2}} \operatorname{erfc}(\gamma)\right\},
\end{gathered}
$$

$$
\begin{aligned}
\delta_{Q}^{(n)}= & 2 \chi^{(n)}\left(1+s_{Q}^{2}\right)-\frac{8}{\pi^{2}} \alpha s_{Q} \int d^{3} q^{\prime} \frac{q_{z}^{\prime 2 n}}{q^{\prime 2}+q_{z}^{\prime 2}} \\
& \times s_{Q^{\prime}} A\left(\left|\vec{q}+\vec{q}^{\prime}\right| R\right) \exp \left(-\frac{q_{z}^{2}+q_{z}^{\prime 2}}{4 \beta^{2}}\right) \cosh \left(\frac{q_{z} q_{z}^{\prime}}{2 \beta^{2}}\right),
\end{aligned}
$$

where in the above, we have defined $\gamma=q / \sqrt{2} \beta$, and

$$
A(q R)=\frac{2}{\left[j_{0,1} J_{1}\left(j_{0,1}\right)\right]^{2}} \int_{0}^{j_{0,1}} d t t J_{0}^{2}(t) J_{0}\left(\frac{q R}{j_{0,1}} t\right)
$$

${ }^{1}$ H. Sakaki, J. Appl. Phys. 19, L735 (1980).

${ }^{2}$ P.M. Petroff, A.C. Gossard, R.A. Logan, and W.W. Wiegmann, Appl. Phys. Lett. 41, 635 (1982).

${ }^{3}$ M. Watt, C.M. Sotomayer-Torres, H.E.G. Arnot, and S.P. Beaumont, Semicond. Sci. Technol. 5, 285 (1990).

${ }^{4}$ R.C. Tonucci, B.L. Justus, A.J. Campillo, and C.E. Ford, Science 258, 783 (1992).

${ }^{5}$ J.P. Leburton, J. Appl. Phys. 56, 2850 (1984).

${ }^{6}$ M.H. Degani and O. Hipólito, Solid State Commun. 65, 1185 (1988).

${ }^{7}$ N.C. Constantinou and B.K. Ridley, J. Phys. Condens. Matter 1, 2283 (1989).
${ }^{8}$ M.H. Degani and G.A. Farias, Phys. Rev. B 42, 11950 (1990).

${ }^{9}$ T. Yıldırım and A. Erçelebi, J. Phys. Condens. Matter 3, 1271 (1991).

${ }^{10}$ T. Yıldırım and A. Erçelebi, J. Phys. Condens. Matter 3, 4357 (1991).

${ }^{11}$ H.-Y. Zhou and S.-W. Gu, Solid State Commun. 91, 725 (1994).

${ }^{12}$ B. Tanatar, J. Phys. Condens. Matter 5, 2203 (1993).

${ }^{13}$ J. Devreese, R. Evrard, E. Kartheuser, and F. Brosens, Solid State Commun. 44, 1435 (1982).

${ }^{14}$ S.I. Pekar, Untersuchungen über die Elekronentheorie der Kristalle (Akademie, Berlin, 1954).

${ }^{15}$ R. Parker, G. Whitfield, and M. Rona, Phys. Rev. B 10, 698 (1974). 